\title{
Evaluation in mammalian oocytes of gene transcripts linked to epigenetic reprogramming
}

\author{
Roberto S Oliveri, Mark Kalisz' ${ }^{1}$ Charlotte Karlskov Schjerling², Claus Yding Andersen, \\ Rehannah Borup ${ }^{2}$ and Anne Grete Byskov
}

Laboratory of Reproductive Biology, The Juliane Marie Centre for Children, Women and Reproduction, Rigshospitalet, DK-2100 Copenhagen, Denmark, ${ }^{1}$ Department of Medical Biochemistry and Genetics, Panum Institute, University of Copenhagen, Rigshospitalet, DK-2100 Copenhagen, Denmark and ${ }^{2}$ RH Microarray Centre, Department of Clinical Biochemistry, Centre of Diagnostic Investigations, Rigshospitalet, DK-2100 Copenhagen, Denmark

Correspondence should be addressed to R S Oliveri; Email: oliveri@rh.dk

\begin{abstract}
The mature mammalian metaphase II (MII) oocyte has a unique ability to reprogram sperm chromatin and support early embryonic development. This feature even extends to the epigenetic reprogramming of a terminally differentiated cell nucleus as observed in connection with somatic cell nuclear transfer. Epigenetic nuclear reprogramming is highly linked to chromatin structure and includes covalent modifications of DNA and core histone proteins as well as reorganization of higher-order chromatin structure. A group of conserved enzymes mediating DNA methylation, methyl-CpG-binding protein (MeCP), histone acetylation and methylation, and chromatin remodeling are extensively involved in epigenetic reprogramming in mammalian cells. Using the oligonucleotide microarray technique, the present study compared the expression levels of $\mathbf{8 6}$ genes associated with epigenetic reprogramming in murine in vivo matured MII oocytes with that of germinal vesicle oocytes. Correlation between biological replicates was high. A total of 57 genes with potential reprogramming effect were detected. In MII oocytes, four genes were significant up-regulated, whereas 18 were down-regulated and 35 unchanged. The significantly regulated genes were validated by real-time quantitative RT-PCR. For example, MII oocytes showed a significant down-regulation of oocytespecific maintenance DNA methyltransferase, Dnmt1o, and up-regulation of MeCP transcript, methyl-CpG binding domain protein 2. Furthermore, histone acetyltransferases were proportionally overrepresented when compared with histone deacetylases. These data elucidate for the first time some of the mechanisms that the oocyte may employ to reprogram a foreign genome either in form of a spermatozoa or a somatic nucleus and may thus be of importance for advancing the fields of stem cell research and regenerative medicine.

Reproduction (2007) 134 549-558
\end{abstract}

\section{Introduction}

The culmination of mammalian oogenesis is the creation of a mature metaphase II (MII) oocyte capable of sustaining fertilization, reprogramming the parental genomes, and supporting early embryonic development. The oocyte cytoplasm rapidly starts to modify the sperm genome upon fertilization resulting in decondensation of paternal chromatin, substitution of protamines with highly acetylated histones, and demethylation of DNA (Santos et al. 2002, Spinaci et al. 2004). By contrast, the oocyte-derived maternal chromatin seems more protected from this extensive reprogramming activity undergoing DNA demethylation at a more passive level during subsequent cleavages (Oswald et al. 2000). The observed asymmetry in parental genome reprogramming is believed to result from marked differences in the original nucleosomal structure of the maternal and paternal chromatin respectively (Mayer et al. 2000, Santos et al. 2002, Spinaci et al. 2004).
Several studies have approached the underlying molecular mechanisms that take place in connection with reprogramming, suggesting that the three-dimensional structure of chromatin is highly important in the regulation of gene expression. Generally, a condensed chromatin structure is associated with an inaccessible and gene repressive state, whereas more decondensed and open chromatin is more amenable to gene activation (Turner 2001).

Notably, transcription levels may be affected by covalent modifications of the DNA helix and core histones comprising the nucleosome, the core unit of chromatin. The complex mechanisms involved in the modifications of chromatin are mediated by a group of highly conserved enzymes, including DNA methyltransferases (DNMTs), methyl-CpG-binding proteins (MeCPs), histone acetyltransferases (HATs), histone deacetylases (HDACs), histone methyltransferases (HMTs), histone demethylases/deiminases (HDMs), and 
chromatin remodeling complexes (ChRs). Covalent DNA methylation, which is the most abundant form of epigenetic modification, occurs at cytosines in the pyrimidine ring of $\mathrm{CpG}$ dinucleotides dispersed throughout the genome and is generally associated with gene silencing. The repressive effect of DNA methylation is mediated by the MeCPs, which again recruit a wide range of co-repressors, including HDACs. Nucleosome core histones are covalently modified at their $\mathrm{N}$-terminal tails, which are highly charged and tightly associated with the DNA helix. In particular, acetylation and methylation have been associated with regulation of gene expression and it has been suggested that the vast number of combinations may actually form a specific histone code, which could determine the binding patterns of regulatory proteins. By contrast, ChRs do not interact covalently with DNA in a sequence-specific manner, but are rather recruited to promoters of target genes by sequence-specific transcription factors. Once recruited, the catalytic subunits of the ChRs (ChRATPases) cause an altered conformation and position of nucleosomes by ATP hydrolysis (Kingston \& Narlikar 1999). This leads to weakened DNA-histone contacts resulting in histone octamers changing positions along the DNA helix (Bazett-Jones et al. 1999, Whitehouse et al. 1999), thus either increasing or reducing the binding of transcription factors to promoter regions. The groups of proteins important to epigenetic modifications and chromatin remodeling do not act independently, but are closely coordinated, often by forming large multiprotein complexes (Nan et al. 1998, Wade et al. 1999, Robertson et al. 2000, Rountree et al. 2000).

Remarkably, the highly specialized ability of the MII oocyte cytoplasm to reprogram a sperm genome extends to a comparable level of dedifferentiation and reprogramming of a diploid somatic nucleus from adult tissue (Wilmut et al. 1997), i.e., somatic cell nuclear transfer (SCNT) or cloning. By contrast, fully grown germinal vesicle (GV) oocytes are not considered capable of adequate reprogramming (Fulka et al. 2002, Tesarik et al. 2003, Zhang et al. 2004). For example, when murine GV oocytes are enucleated and the remaining ooplast subsequently is used for SCNT, nuclear reprogramming is severely reduced, without formation of pseudo-pronuclei or cleavage (Gao et al. 2002). The factors responsible for gamete and somatic reprogramming are likely to become available during the final stages of oogenesis during the transition from GV to MII stage, thus highlighting the MII oocyte cytoplasm as the key player in the reprogramming of a specialized genome into a totipotent state. However, the exact mechanisms by which the MII oocyte cytoplasm erases the established patterns of gene expression imposed on the sperm genome or a somatic nucleus remain largely unknown.

To elucidate some of the tools involved in early embryonic reprogramming, the levels of gene transcripts believed to be of importance to epigenetic modifications and chromatin remodeling were detected by oligonucleotide microarrays in in vivo matured MII oocytes and compared with fully in vivo grown GV oocytes. Furthermore, real-time quantitative RT-PCR (qRT-PCR) was used to validate expression patterns of a number of significantly regulated reprogramming genes.

\section{Results \\ Oocyte collection and validation of RNA}

On average, 320 and 421 oocytes were pooled for each biological replicate of GV and MII oocytes respectively. An examination of total RNA on a 2100 Bioanalyzer confirmed the extraction of high-quality RNA (data not shown). Correlations between biological replicates were substantially high, indicating identical global transcription patterns and thus high reliability of the microarray gene chip procedure performed on minute amounts of oocyte RNA (Fig. 1 and Table 1). An unsupervised hierarchical cluster analysis using all detected probe sets showed that biological replicates representing the same developmental stage were independently grouped together as expected. Genes known to be preferentially expressed in oocytes such as the zona pellucida glycoproteins $(Z p 1, Z p 2$, and $Z p 3$ ), growth differentiation factor 9 (Gdf9), bone morphogenetic protein 15 (Bmp15), moloney sarcoma oncogene (Mos), cyclin B1 (Ccnb1), spindlin (Spin), MATER (Nalp5), zygote arrest 1 (Zar1), STELLA (Dppa3), and oct4 (Pou5f1) were abundantly detected on the chip set (Supplementary Table 1, which may be viewed online at www.reproduction-online.org/supplemental). Although

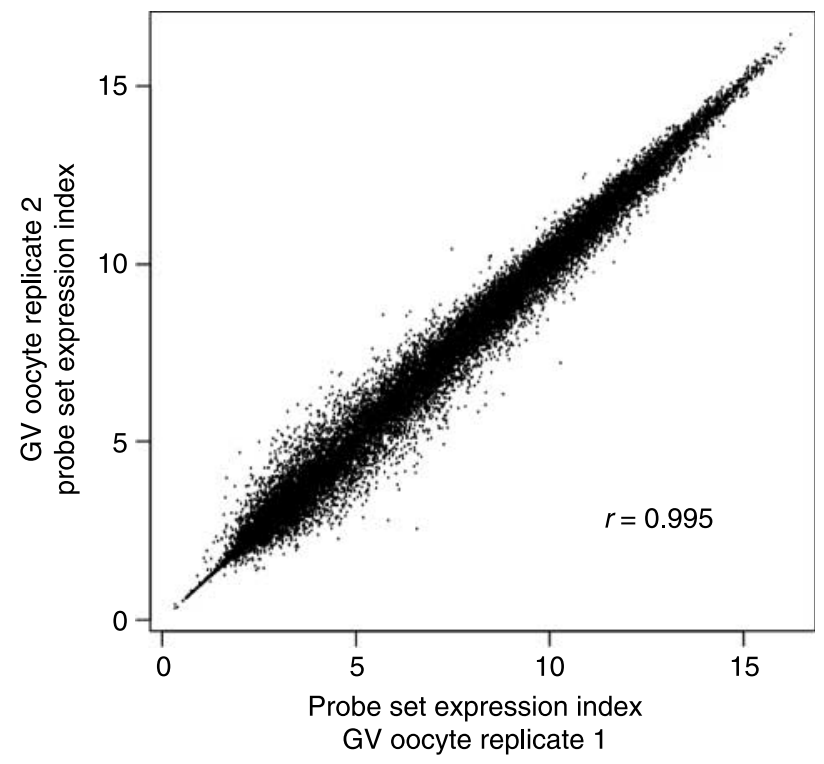

Figure 1 Correlation plot illustrating the concordance in probe set expression values $(n=45101)$ between two of the biological replicates. The high correlation coefficient $(r)$ confirms the reliability of Affymetrix gene chip technology performed on minute amounts of mRNA extracted from mammalian oocytes. 
Table 1 Characteristics for microarray chip samples. A high correlation coefficient $(r)$ between biological replicates indicates almost identical global transcription patterns and thus high validity and reliability of the microarray procedure per se performed on minute amounts of RNA.

\begin{tabular}{lccc}
\hline $\begin{array}{l}\text { Develop- } \\
\text { mental stage }\end{array}$ & $\begin{array}{c}\text { Biological } \\
\text { replicates }(\boldsymbol{n})\end{array}$ & $\begin{array}{c}\text { Median (range) } \boldsymbol{r} \\
\text { between biological } \\
\text { replicates }\end{array}$ & $\boldsymbol{P \text { value }}$ \\
\hline GV oocytes & 3 & $0.993(0.992-0.995)$ & $<0.0001$ \\
MII oocytes & 5 & $0.972(0.955-0.992)$ & $<0.0001$
\end{tabular}

GV, germinal vesicle; MII, metaphase II.

oocytes underwent careful denudation, we checked for potential contamination of heterolog RNA from cumulusgranulosa cells. The absence of cumulus transcripts such as hyaluronic synthase 2, pentraxin 3, and gremlin 1 confirmed the oocyte homogeneity of the collected samples.

\section{Global oocyte gene expression and inclusion of genes for further analyses}

After excluding probe sets that were $100 \%$ absent in both groups of replicates, the data were reduced from 45101 to 24919 probe sets. To minimize false positives, a probe set was then included for further analysis provided that a $100 \%$ present call was found in either one of the two groups of replicates, irrespective of its call percentage in the other replicate group. Accordingly, a total of 14697 probe sets were expressed exclusively in GV oocytes, in MII oocytes, or both. A $t$-test for difference in expression level between GV and MII oocytes Benjamini-Hochberg corrected for multiple testing yielded that 5169 (35\%) probe sets were significantly down-regulated, $609(4 \%)$ probe sets were up-regulated, and 8919 (61\%) probe sets were equally expressed. A complete list of up- and down-regulated genes is available at http://www.ebi.ac. uk/arrayexpress/ (accession no. E-MEXP-1146).

\section{General characteristics of reprogramming genes}

A comprehensive list of 86 candidate transcripts involved in epigenetic modifications and chromatin remodeling was constructed from available literature and the Nuclear Protein Database (http://npd.hgu.mrc.ac.uk. Accessed April 2, 2006). Supplementary Table 1 (which may be viewed online at www.reproduction-online.org/supplemental) shows the average expression indices, fold changes, and corrected $P$ values supplemented with values for a number of transcripts preferentially expressed in the oocyte. In total, 57 genes (70\%) were detected in GV and/or MII oocytes on the chip set. Within the 33 genes detected as down-regulated, 14 had fold changes from -1 to -2 , eight had fold changes from -2 to -5 , three had fold changes from -5 to -10 , and eight had fold changes $<-10$. A total of 24 genes were detected as up-regulated with 16 genes having fold changes from 1 to 2 and eight genes having fold changes ranging from 2 to 5 . However, when only statistically significant fold changes were considered, four genes were up-regulated and 18 genes were down-regulated, whereas 35 genes indicated an unchanged level of gene expression. The dendrogram in Fig. 2 illustrates the final clustering tree, where significantly regulated genes close to each other have high similarity in their standardized expression values across all eight samples.
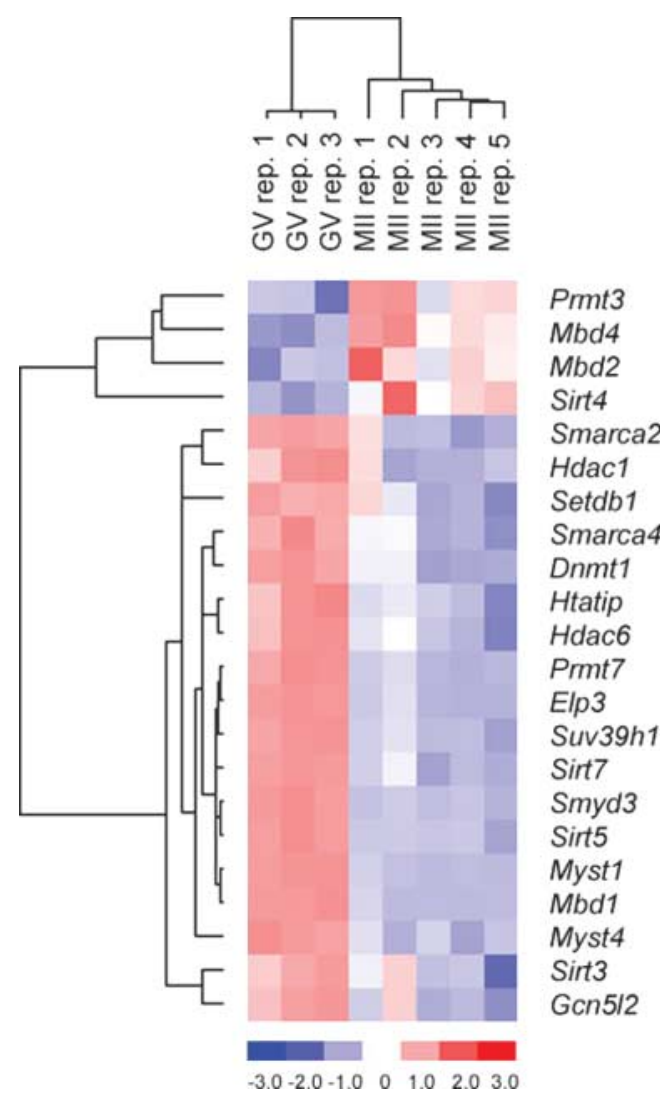

Figure 2 Dendrogram illustrating the final hierarchical clustering tree of 22 significantly up- or down-regulated reprogramming genes (for gene symbols, see Supplementary Table 1, which may be viewed online at www.reproduction-online.org/supplemental) in MII oocytes when compared with GV oocytes (five and three biological replicates respectively). The expression values for a gene across all eight samples are standardized to have mean 0 and S.D. 1 by linear transformation. The distance between two genes is defined as $1-r$, where $r$ is the standard correlation coefficient between the eight standardized values of two genes. Two genes with the closest distance are first merged into a supergene, connected by branches with length representing their distance, and deleted for future merging. The expression level of the newly formed supergene set is the average of standardized expression levels of the two genes (average linkage) for each sample. Then, the next pair of genes (supergene) with the smallest distance is chosen to merge and the process is repeated until all genes are merged into one final cluster. Red and blue color indicates up- and down-regulation respectively relative to the mean expression of the eight samples combined. 


\section{DNMTs and MeCPs}

The gene of the oocyte variant form of the maintenance DNMTs, Dnmt1o, was abundantly expressed in GV oocytes and then significantly down-regulated in MII, whereas both the de novo DNMTs Dnmt3a and Dnmt3b indicated unchanged level of expression. Of the seven known MeCPs that bind to methylated DNA, two were significantly up-regulated $(\mathrm{Mbd} 2, \mathrm{Mbd} 4)$, one was down-regulated (Mbd1), one (Kaiso/Zbtb33) showed no change, while Mecp2, Zbtb4, and Zbtb38 were not detected on the chip.

\section{HATs}

HATs can be classified into five subgroups according to sequence homology and internal protein domains. All five members of the MYST subgroup were detected in oocytes. TIP60 (Htatip), MOF (Myst1), and MORF (Myst4) showed significant down-regulation, while HBO1 (Myst2) and MOZ (Myst3) were unchanged. Of the members belonging to the GNAT subgroup, elongation protein 3 homolog (Elp3), and GCN5 (Gcn5/2) were significantly downregulated, histone aminotransferase 1 (Hat1) was unchanged and p300/CBP-associated factor (Pcaf) was absent. The two transcriptional 'coactivators' E1A-binding protein p300/p300 (Ep300) and CREB-binding protein/CBP (Crebbp), which are almost functionally equivalent (p300/CBP subgroup), were both expressed in oocytes at an unchanged level as were the three nuclear hormone-receptor cofactors (SRC subgroup) SRC-1 (Ncoa1), GRIP1 (Ncoa2), and ACTR (Ncoa3). The basal transcription factors $\mathrm{TAF}_{\mid I} 250$ (Taf1) and TFIIIC (Gtf3C) were also expressed at an unchanged level. The more recently identified HATs, Cdyl, Atf2, and Ogt were all expressed in oocytes at an unchanged level.

\section{HDACs}

HDACs are subdivided into four classes according to phylogenesis. Class I members Hdac1, Hdac2, and Hdac 3 were expressed in oocytes, whereas $\mathrm{Hdac} 8$ was absent. Hdac1 was down-regulated and Hdac2 and $\mathrm{Hdac} 3$ were unchanged. No class II HDAC was detected, except Hdac6 and Hdac9, which were down-regulated and unaffected respectively. Except Sirt2, all sirtuins or class III HDACs were expressed in oocytes of which Sirt4 was up-regulated, Sirt3, Sirt5, and Sirt7 were downregulated, and Sirt1 and Sirt6 showed no change. The single class IV member, Hdac11, was not detected.

\section{HMTs and HDMs}

HMTs either activate or repress gene function depending on the residue of the modified amino acid. Histone methylation at positions $\mathrm{H} 3 \mathrm{~K} 4, \mathrm{H} 3 \mathrm{~K} 36$, or $\mathrm{H} 3 \mathrm{~K} 79$ is associated with gene activation. Of the activating HMTs,
DOT1-like (Dot11) was undifferentially expressed, SET and MYND domain containing 3 (Smyd3) was downregulated, and SET domain containing 1A (Setd1a), myeloid/lymphoid or mixed-lineage leukemia 1 (MII1), SET7/9 (Setd7), and Meisetz PR domain containing 9 (Prdm9) were absent. Of the HMTs associated with repression (H3K9, H3K27, and H4K20), suppressor of variegation 3-9 homolog 1 (Drosophila) (Suv39h1) and ESET SET domain, bifurcated 1 (Setdb1) were downregulated; SUV39h2 (Suv39h2), Eu-HMTase1/GLP1 (Ehmt1), ENX-1 (Ezh2), PR-SET7/SET8 (Setd8), SUV420h1 (Suv420h1), SUV4-20h2 (Suv420h2), and RIZ (Prdm2) were unchanged; and G9a (Ehmt2) was absent. NSD1 (Nsd1), which can act both as activator and repressor, was not detected.

Of the arginine HMTs, one was slightly up-regulated (Prmt3), one was down-regulated (Prmt7), two were undifferentially expressed (Prmt1, Prmt2), and three were absent (Prmt4, Prmt5, and Prmt6).

All currently known HDMs were absent in oocytes, except lysine-specific demethylase 1 (Aof2), which was expressed at an unchanged level.

\section{ChR-ATPases}

The ChR-ATPases are classified into subfamilies according to the presence of conserved domains outside their catalytic region. The two bromodomain SNF2-subfamily ATPases hBRM (Smarca2) and BRG1 (Smarca4) were both expressed in oocytes and down-regulated. The SANT domain ISWI-subfamily members SNF2L (Smarca1) and SNF2H (Smarca5) were absent or unchanged respectively. Among the four chromodomain CHD-subfamily ATPases, only $\mathrm{Mi}-2 \beta$ (Chd4) was expressed at unchanged level, while Chd1, Chd2, and Mi-2 $\alpha$ (Chd3) were absent.

\section{Real-time quantitative RT-PCR}

The four transcripts having statistically significant up-regulation and the 18 transcripts being downregulated as indicated by the present microarray data were selected for further analysis by real-time quantitative RT-PCR. To select an appropriate internal reference gene, we tested the expression of the three housekeeping genes $\beta$-actin (Actb), porphobilinogen deaminase $(\mathrm{Hmbs})$, and TATA box-binding protein (Tbp) using equivalent amounts of total RNA converted into cDNA. A statistically insignificant relationship existed between oocyte developmental stage and expression of $A c t b$, which was confirmed by using NormFinder. Accordingly, Actb was used as internal reference gene for further analyses.

The RT-PCR expression data for the 22 reprogramming gene transcripts are presented in Fig. 3. The absence of 


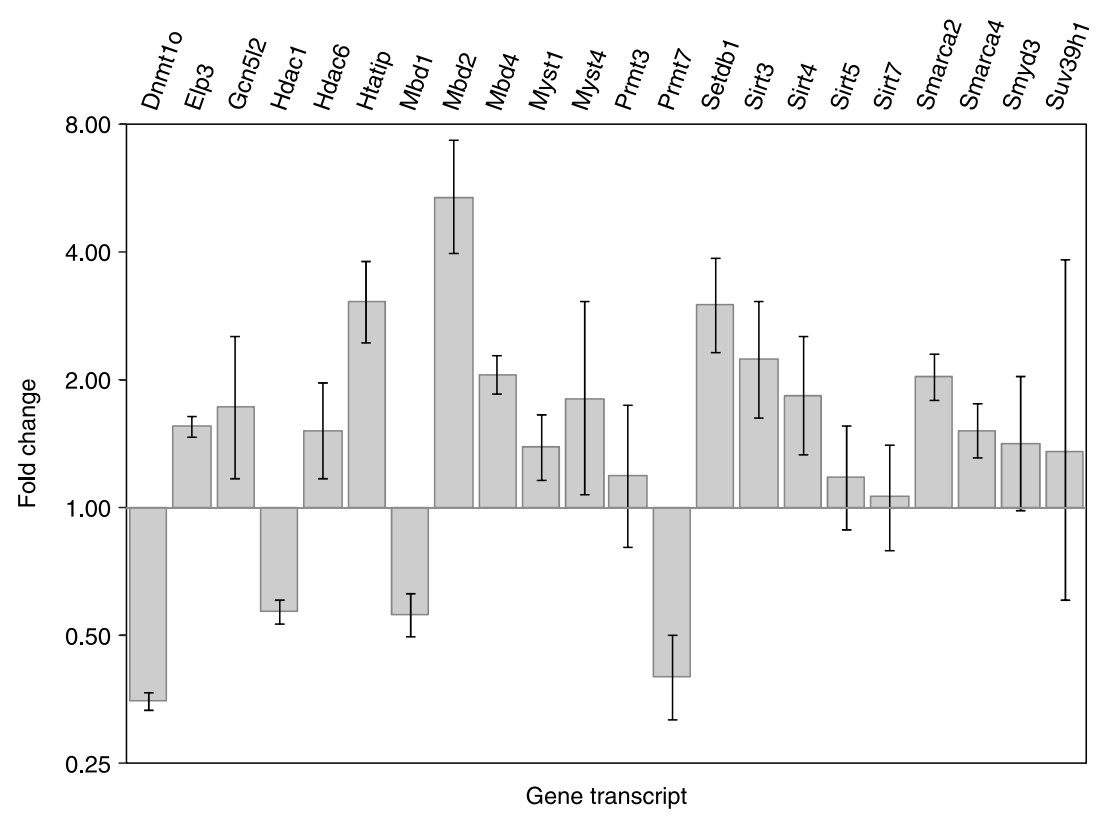

Figure 3 Bar chart showing fold changes of 22 reprogramming genes measured by real-time quantitative RT-PCR. Mean fold changes are expressed on a $\log _{2}$ scale as metaphase II oocyte values relative to germinal vesicle stage oocytes. Whiskers represent 95\% confidence intervals. non-specific amplified products was confirmed by melting curve analysis. When comparing RT-PCR fold changes with the microarray data, we observed some discrepancy regarding direction and amplitude. To test whether such differences could be explained by the use of random hexamer primers during cDNA synthesis, we repeated the RT-PCR using oligo(dT) primers (Fermentas, Helsingborg, Sweden). In this case, there was a significantly higher correlation between array data and qRT-PCR data regarding direction and amplitude, suggesting the occurrence of selective deadenylation during meiotic maturation.

\section{Discussion}

To our knowledge, this is the first study that specifically investigates the expression levels of a larger group of genes currently believed to be involved in epigenetic modifications and chromatin remodeling. We show that 57 out of these 86 gene transcripts are present in mouse oocytes. We also validated 22 reprogramming gene transcripts that indicated statistically significant up- or down-regulation from GV to MII stage using real-time quantitative RT-PCR. Of particular interest, we observed that the oocyte-specific maintenance DNMT, Dnmt1o, is down-regulated and that the $M b d 2$ gene showed a significant fivefold up-regulation. Furthermore, we observed that HATs were proportionally overrepresented when compared with the antagonistic HDACs. Taken together, these data suggest that the reprogramming machinery of the oocyte correlates with developmental state in late oogenesis.

In SCNT, the success of epigenetic nuclear reprogramming is thought to depend on tightly coordinated successive events (Gurdon 2006). First, the epigenetic constraints once imposed on the foreign nucleus need to be erased. Active genes composing the phenotype must be repressed, whereas inactive or repressed genes must become accessible to regulatory protein complexes. Similar to the sperm genome, these changes include decondensation of higher-order chromatin structure, covalent histone modifications, and DNA demethylation. Subsequently, genes required for early embryonic development need to be correctly imposed on the genome and expressed, including stem cell genes associated with pluripotency (Simonsson \& Gurdon 2004, Gurdon 2006).

During oocyte maturation, we observed a significant down-regulation of Dnmt1o in both array data and qRTPCR, which is consistent with earlier observations (Ratnam et al. 2002, Vassena et al. 2005). This oocytespecific form has a nuclear localization at the earliest stages of oocyte growth, but then becomes localized in a cytoplasmic shell just within the GV oocyte plasma membrane prior to ovulation (Carlson et al. 1992). It remains here - potentially without effect on the chromatin - during pre-implantation development except at the eight-cell stage where it briefly enters and exits the nuclei, perhaps to maintain methylation patterns of imprinted genes (Carlson et al. 1992, Mertineit et al. 1998). Maternal Dnmt1o re-enters the nucleus after implantation and is soon replaced by its somatic counter form, Dnmt1s, expressed by the embryonic genome. The nuclear absence of Dnmt1o during late oogenesis and early embryonic development is in accordance with the lack of maintenance methylation and hence global hypomethylation of zygotic genome during early cleavage. The attenuated 
transcription of Dnmt1o observed during meiotic maturation suggests that the cytoplasmic storage reaches adequate levels in the fully grown GV oocyte. The poor success rates and developmental abnormalities observed in offspring derived by SCNT may be attributed to the counteracting presence of Dnmt1s in somatic nuclei used as donors. Notably, the rapid genome-wide DNA demethylation of the paternal genome observed within hours after fertilization is believed to be an active process and not a result of nuclear lack of Dnmt1o, since it occurs before the first DNA replication and cleavage in the newly formed zygote (Oswald et al. 2000). Thus, DNA methylation is a reversible process, which strongly indicates the presence of specific demethylases residing in the mature MII cytoplasm.

The identity of specific DNA demethylases responsible for paternal genome reprogramming has remained a subject of controversy. Similar to methylated DNA, methylation of core histones was previously considered to be irreversible, but a growing number of histone demethylases/deiminases have recently been identified and characterized (Shi et al. 2004, Cloos et al. 2006). Among the reprogramming genes yielding up-regulation in MII oocytes, we observed that Mbd2 yielded a particularly high fold change in comparison to the small fold changes otherwise observed. Notably, meiotic maturation is characterized by general transcriptional silence and considerable mRNA degradation. Earlier, Szyf and co-workers reported to have purified a bona fide demethylase able to catalyze active demethylation by hydrolytic cleavage releasing the methyl group from cytosine in the form of methanol (Ramchandani et al. 1999). The enzyme was suggested to be identical to Mbd2 (Bhattacharya et al. 1999, Cervoni \& Szyf 2001, Detich et al. 2002), and has shown to be processive (Cervoni et al. 1999), a critical feature for the global demethylation event following fertilization. It has also been reported that overexpression of $\mathrm{Mbd} 2$ results in histone acetylation and global demethylation in nontransformed NIH 3T3 cells and cancer cell lines (D'Alessio \& Szyf 2006). However, other investigators have been unable to demonstrate a demethylating activity of $\mathrm{Mbd} 2$. In fact, $\mathrm{Mbd} 2$ was reported to associate with HDAC1 in the gene repressing MECP1 complex ( $\mathrm{Ng}$ et al. 1999) and, importantly, Mbd2-deficient oocytes retain the capacity to demethylate paternal genomes at the same level as wild-type controls (Santos et al. 2002). The latter findings suggest that Mbd2 does not have a DNA demethylating effect in early embryonic development or that other compensatory mechanisms may exist. However, an alternative explanation might be the presence of an as yet unidentified oocyte-specific Mbd2 transcript ('Mbd2o') accounting for the demethylating activity. Oocyte-specific isoforms have been reported for other factors involved in chromatin remodeling (Mertineit et al. 1998, Tanaka et al. 2001), and a truncated alternatively spliced Mbd2 transcript exists in the testis (Hendrich \& Bird 1998).

The discovery that methylated DNA causes its gene repressive effect through the binding of MeCPs and recruitment of chromatin-condensing HDAC complexes (Nan et al. 1998), initiated the view that the relationship between methylated DNA and histone modifications is unidirectional. However, a growing body of evidence suggests that the relationship between chromatin structure and DNA methylation may be more bidirectional than previously thought and that chromatin itself can influence the methylation status of DNA (D'Alessio \& Szyf 2006, Milutinovic et al. 2006). For example, the HDAC-inhibitor sodium butyrate can trigger replicationindependent DNA demethylation (Szyf et al. 1985) and inhibitors of histone acetylation inhibit DNA demethylation (Cervoni et al. 2002). Histone acetylation induced by external mediators, including HDAC inhibitors, initiates demethylation of ectopically methylated genes, suggesting that histone acetylation precedes DNA demethylation (Cervoni \& Szyf 2001, Detich et al. 2003). Interestingly, it has been demonstrated that HAT activity is associated with Mbd2 in vivo (Angrisano et al. 2006) and that Mbd2 co-localizes with the HAT, CBP (Crebbp) (D'Alessio \& Szyf 2006). We observed that 18 out of 19 known HATs, including Crebbp, were abundantly expressed in oocytes, as opposed to 11 out of the 18 known HDAC members. We hypothesize that a yet unidentified oocyte-derived active DNA demethylase, which acts during fertilization, may be facilitated by a cellular environment characterized by the preponderant presence of HATs.

Among the ChR-ATPases, BRG1 (Smarca4) attracts particular interest. Our array data indicated downregulation of Smarca4 in MII oocytes, whereas qRTPCR indicated unchanged expression or slight increase. The apparent presence of BRG1 in mature oocytes confirms that BRG1 plays an important role in early embryonic development, since BRG1-depleted oocytes are arrested at the two-cell stage in mice (Bultman et al. 2006). Further depletion of BRG1 has been shown to inhibit the reprogramming capacity in egg cell extracts, while its overexpression is associated with extended reprogramming activity (Hansis et al. 2004).

Our data revealed that $\sim 14000$ probe sets were detected in oocytes, which is a number very similar to that found by others using Affymetrix oligonucleotide microarray gene chips on oocytes (Pan et al. 2005). Global expression profiles between the biological replicates of GV oocytes were very similar, while those between MII replicates were slightly more varied. Such differences in similarity between MII populations comply with observations seen in human oocytes (Goto et al. 2002), and may be attributed to the general mRNA degradation during meiotic maturation.

The detection of an abundant expression of oocytespecific genes combined with the absence of cumulus 
granulosa cell transcripts increased the validity of the microarray procedure performed on minute amounts of mammalian oocyte RNA. Considerably high correlation coefficients and group clustering between biological replicates supports experimental reliability. Furthermore, we aimed at optimizing microarray data processing by implementing quantile normalization method, which is especially suitable for pairwise comparisons (Bolstad et al. 2003). We also used the GCRMA method to calculate gene expression indices, since it has less variance at low RNA concentrations, better precision for lower expression values, and more consistent estimates of fold change when compared with other normalization models (Irizarry et al. 2003a).

Validation of 22 reprogramming genes by RT-PCR confirmed the presence of reprogramming gene transcripts in mammalian oocytes. However, discordance in some cases was observed when results were compared with the microarray data. These differences are likely to be attributed to the use of different types of primers during the first-strand synthesis in the RT step. Following standard protocols, T7-oligo(dT) primers were used in the microarray experiments, whereas random hexamer primers were used for RT-PCR. During mammalian oocyte maturation, some poly(A) mRNAs are deadenylated, but not degraded (El Mouatassim et al. 1999, Lequarre et al. 2004). The subsequent translation of mRNA is linked to the extent and timing of its adenylation status during the transition from GV to MII oocyte stage. Accordingly, the use of oligo(dT) primers for RT may increase the risk that some deadenylated transcripts with a too short or completely absent poly $(\mathrm{A})$ tail will not be detected in an equal abundance in MII oocytes, while this should not affect the binding of random hexamer primers. Accordingly, the apparent down-regulation in the array data for some gene transcripts may therefore represent deadenylation instead of degradation. This particular outcome has been verified in a study comparing the use of random hexamer and oligo(dT) primers in mammalian oocytes undergoing meiotic maturation (Lequarre et al. 2004). When the RT-PCR procedure was repeated using oligo(dT) primers, we also observed a significantly higher agreement between microarray and RT-PCR data. Nonetheless, some inconsistency in reproducibility is still to be expected due to statistical variation at low copy numbers for genes that are expressed at very low initial amounts (Peccoud \& Jacob 1996). Currently, no validated method exists for the adequate quantification of mammalian MII oocyte transcripts using microarrays. Even with the implementation of specific primers that initiate cDNA synthesis at multiple steps along the mRNA during the Affymetrix amplification steps, some discordance is still observed between microarray and RT-PCR data (Su et al. 2007).
In conclusion, a total of 57 out of 86 known genes of importance to epigenetic modifications and chromatin remodeling were detected in murine oocytes. Taken together, these observations support the notion that the reprogramming machinery is fully functional in the mature MII oocyte, possibly including specific active DNA demethylating activity facilitated by preceding HAT activity. However, the precise extent of the translation of these transcripts remains to be evaluated since transcription rates do not necessarily correlate with protein activity. The scarcity of biological material seriously impedes proteomic investigations in mammalian oocytes at present.

The presented list of reprogramming genes may be useful for future investigations in areas involving epigenetic modifications, such as developmental biology and regenerative medicine.

Identifying key oocyte factors involved in epigenetic modifications and chromatin remodeling will provide further insight into how the highly differentiated parental genomes are restored to totipotency. These profound rearrangements are likely to be similar to the reprogramming event observed during SCNT. Hence, the characterization of one or more dedifferentiating 'master genes' may have important implications for human stem cell research, ultimately providing a route towards autologous cell replacement without the need for human eggs or embryos (Oliveri 2007).

\section{Materials and Methods Collection of mouse oocytes}

Oocytes were obtained from 3-week-old B6D2F1 mice (Taconic Europe, Ejby, Denmark) and were carefully processed to avoid human contamination. Mice were handled under standard laboratory conditions and experiments were conducted following national guidelines on animal care (Danish Ministry of Justice; approval 2003/561-713).

For the collection of GV oocytes, mice were each injected with 15 IU menotropin (Menopur; Ferring, Copenhagen, Denmark) and killed by cervical dislocation $42 \mathrm{~h}$ later. Ovaries were isolated and antral follicles were punctured by 27-ga needles. Cumulus-enclosed GV oocytes were released into $\alpha$-MEM (Invitrogen) supplemented with $4 \mathrm{mmol} / \mathrm{l}$ hypoxanthine (Sigma) to inhibit GV breakdown during handling. Only spherical GV oocytes with a distinct GV and attached cumulus cells were collected and immediately underwent mechanical denudation with a fine bore glass pipette.

For the collection of MII oocytes, mice were first primed with $15 \mathrm{IU}$ menotropin (Menopur; Ferring) followed by injection of $5 \mathrm{IU}$ of choriongonadotropin $44 \mathrm{~h}$ later (Pregnyl; Organon, Oss, The Netherlands), and were then killed $15 \mathrm{~h}$ later. Collected oviducts were punctured in $\alpha$-MEM to yield the cumulus-oocyte complexes (COCs). COCs were briefly exposed to $80 \mathrm{IU} / \mathrm{ml}$ hyaluronidase (SynVitro Hyadase; MediCult, Jyllinge, Denmark) at $37^{\circ} \mathrm{C}$ to remove attached 
cumulus granulosa cells. Only spherical MII oocytes with a distinct first polar body were selected.

After denudation, all oocytes were briefly exposed to $10 \mathrm{mg} / \mathrm{ml}$ protease (Sigma) at $37^{\circ} \mathrm{C}$ to remove the zona pellucida and remaining attached cumulus cell debris. Oocytes were washed several times and collected for immediate RNA extraction.

\section{RNA extraction and quality assessment}

Oocyte RNA was obtained using the RNeasy Micro Kit (Qiagen), which enriches mRNAs from small samples by selectively excluding shorter RNAs such as rRNAs and tRNAs. Briefly, pools of oocytes were lysed with guanidine isothiocyanate-containing buffer and were applied to spin columns for adsorption of the RNA. Carrier was included to improve the yield of low-abundance mRNAs further (Ståhlberg et al. 2004). Pellets were washed with $80 \%$ ethanol, centrifuged, and airdried. Subsequently, the RNA was eluted in nuclease-free water and immediately stored at $-80{ }^{\circ} \mathrm{C}$ until use.

To ensure that high-quality RNA was hybridized to the gene expression arrays, isolated RNA was assessed on a 2100 Bioanalyzer using a RNA 6000 Pico LabChip (Agilent Technologies, Palo Alto, CA, USA).

The protocol used for microarray chip hybridization required at least $100 \mathrm{ng}$ total RNA for each biological replicate. Due to limited sample quantities of oocytes, pooling of total RNA from repeated experiments was necessary, so with an expected average amount of $0.3-0.5 \mathrm{ng}$ total RNA in a murine oocyte, each replicate was constructed from pooled RNA of oocytes from $\sim 20$ mice. We isolated sufficient oocyte RNA for the generation of three and five biological replicates of GV and MII oocytes respectively.

\section{Amplification and hybridization to microarray chips}

Due to the small amounts of initial RNA, the process required an amplifying two-cycle target labeling assay step to obtain sufficient amounts of labeled cRNA target for analysis with arrays. A minimum of $100 \mathrm{ng}$ total RNA was used to synthesize double-stranded cDNA with the Superscript Choice system (Invitrogen) with an oligo(dT) primer containing a T7 RNA polymerase promoter (GenSet). The cDNA was used as the template for an in vitro transcription reaction to synthesize antisense cRNA (BioArray high yield RNA transcript labeling kit; Enzo, Farmingdale, NY, USA). The signal was then amplified in a second cDNA synthesis step using random primers for firststrand synthesis and T/-oligo(dT) primer for the second-strand synthesis. The amplified cDNA was used as template for a second in vitro transcription to synthesize biotin-labeled antisense cRNA (BioArray high yield RNA transcript labeling kit; Enzo).

The labeled cRNA was fragmented at $94{ }^{\circ} \mathrm{C}$ for $35 \mathrm{~min}$ in fragmentation buffer $(40 \mathrm{mM}$ Tris, $30 \mathrm{mM}$ magnesium acetate, and $10 \mathrm{mM}$ potassium acetate) and was hybridized for $16 \mathrm{~h}$ to the Affymetrix GeneChip Mouse Genome 4302.0 Array (Affymetrix, Santa Clara, CA, USA), which covers transcripts and variants from 34000 well-characterized mouse genes. Probe sets on this array are derived from sequences from
GenBank, dbEST, and RefSeq. The arrays were washed and stained with phycoerythrin-streptavidin using a Fluidics Station 450 (Affymetrix), and the arrays were scanned in a GeneArray 3000 scanner (Affymetrix). Data were digitalized and converted into CEL-format (v. 3) for data analysis.

\section{Microarray data analyses}

To adjust the overall chip brightness of the arrays to a similar level and thus make the individual chips comparable with each other, the quantile normalization method was used (Bolstad et al. 2003). To summarize the probe level data for each probe set, an expression index was calculated by the guaninecytosine corrected robust multiarray analysis (GCRMA) method (Irizarry et al. 2003b). Expression values were estimated on logarithmic scale (base 2) instead of raw intensities because of the variance-stabilizing effect of this transformation.

To remove the ambiguity of multiple and less specific probe sets, we retained only transcripts with an Affymetrix oligonucleotide probe set corresponding to a single gene ('_at' suffices) or with anticipated oocyte-specific splice variants ('_a_at' suffices) only allowing less specific probe sets if a unique probe set for a gene was missing on the gene chip at the time of chip manufacturing. Probe sets were then cross-examined in the ADAPT database (Leong et al. 2005) to ensure that only probe sets representing mRNAs remained, thus avoiding regions outside exons and $3^{\prime}$ UTR. Eventually, if a gene was represented by more than one unique probe set, we selected the probe set with most present calls or positioned in the most distal $3^{\prime}$ end of the transcript.

Hierarchical clustering was performed only on genes showing statistically significant up- or down-regulation as identified by $t$-test and with a $P$ value corrected for multiple testing with the Benjamini-Hochberg method to avoid erroneous result by less variant or absent genes (Benjamini \& Hochberg 1995). Significance level was 0.05 , distance metric was $1-r$, and linkage method was centroid. To validate the overall quality of the experiment, hierarchical clustering was also performed on sample level, since biological replicates representing the same condition should to be grouped together because of identical global expression pattern. Accordingly, they should be separated from samples representing other conditions.

Normalization and calculation of gene expression indices were performed with the GCRMA package (Wu et al. 2004), comparisons of samples were performed with the simpleaffy package (Wilson \& Miller 2005), and Benjamini-Hochberg corrected $P$ values were calculated using the multtest package (Pollard et al. 2004). All packages were run in the $R$ statistical software (http://www.r-project.org). Hierarchical clustering was performed in DNA-Chip Analyzer (dChip) 2005 ( $\mathrm{Li}$ \& Wong 2003). Microarray analyses are reported according to the MIAME statement (Brazma et al. 2001).

\section{Real-time quantitative RT-PCR}

Oocytes were collected and total RNA was extracted as described above. cDNA was synthesized with Superscript III (Invitrogen) or iScript cDNA Synthesis Kit (Bio-Rad Laboratories) using oligo(dT) or random hexamers as first-strand 
primer. Equal amounts of total RNA (24 ng) were converted into cDNA for GV and MII samples respectively. Subsequently, the cDNA elute was diluted 1:20 before downstream application.

For PCR amplification, the gene-specific primers were constructed using Primer3 (Rozen \& Skaletsky 2000) or PrimerBank (Wang \& Seed 2003). Primers were carefully chosen or designed to eliminate the risk of amplifying unspecific targets, such as primer dimers or genomic DNA. Primer sequences, exon complementarity, melting temperature, GenBank accession number, and size of the amplified product are presented in Supplementary Table 2, which may be viewed online at www.reproduction-online.org/supplemental. Expression levels were quantified in real time on a LightCycler Instrument (Roche Diagnostics) using LightCycler FastStart DNA Master PLUS SYBR Green I (Roche Applied Science) or SYBR Premix Ex Taq kit (Takara, Madison, WI, USA) as detection chemistry. The PCR mixture consisted of $2 \mu$ diluted CDNA, $1 \mu$ l gene-specific primers, and 2 or $5 \mu$ l SYBR Green I master mix (depending on manufacturer), and added water to a total volume of $10 \mu \mathrm{l}$. First, cDNA was denatured and preincubated by heating for $10 \mathrm{~s}$ at $95^{\circ} \mathrm{C}$. Template was then amplified by 45 cycles consisting of denaturation for $5 \mathrm{~s}$ at $95^{\circ} \mathrm{C}$, annealing for $20 \mathrm{~s}$ at $55^{\circ} \mathrm{C}$, and extension for $10 \mathrm{~s}$ at $72{ }^{\circ} \mathrm{C}$. At least three replicates were used for each reaction, and a minus RT and a minus template served as negative controls. To verify RT-PCR product identity, melting point curves were generated following amplification. Samples were heated to $95^{\circ} \mathrm{C}$ followed by immediate cooling for $15 \mathrm{~s}$ at $65^{\circ} \mathrm{C}$ and then slowly heating by $0.1{ }^{\circ} \mathrm{C} / \mathrm{s}$ up to $95^{\circ} \mathrm{C}$, while monitoring fluorescence continuously.

To determine the effect of meiotic maturation on the expression of a candidate internal reference gene, we evaluated a range of housekeeping genes. Briefly, identical amounts of input RNA from GV and MII oocytes were used for each cDNA reaction. Putative candidate reference genes were amplified and the gene showing the lowest variability was chosen as internal reference using NormFinder (Andersen et al. 2004).

All target genes were normalized against the chosen internal reference gene within the log-linear phase of the amplification curve, and relative quantification was assessed using the $2^{-\Delta \Delta C_{T}}$ method, where $\Delta \Delta C_{\mathrm{T}}=\left(C_{\mathrm{T} \text {-target }}{ }^{-} C_{\mathrm{T} \text {-reference }}\right)_{\mathrm{MII}}-\left(C_{\mathrm{T} \text {-target }}{ }^{-} C_{\mathrm{T}-}\right.$ reference) $)_{\mathrm{GV}}$ (Pfaffl 2001). All fold changes are presented as means with $95 \%$ confidence intervals.

\section{Acknowledgements}

We thank Drs Aashir Awan and Christian Olesen for assistance with RNA extraction and helpful discussions, and technician Tiny Roed for assistance with animal care. This work was supported by The Danish Medical Association Research Fund and The Leo Nielsen and Karen Margrethe Nielsen Foundation for Basic Medical Research. R S Oliveri is supported by a research fellowship grant from The Faculty of Health Sciences, University of Copenhagen. Disclosures: the authors declare no potential conflicts of interest.

\section{References}

D'Alessio AC \& Szyf M 2006 Epigenetic tete-a-tete: the bilateral relationship between chromatin modifications and DNA methylation. Biochemistry and Cell Biology 84 463-476.

Andersen CL, Jensen JL \& Ørntoft TF 2004 Normalization of real-time quantitative reverse transcription-PCR data: a model-based variance estimation approach to identify genes suited for normalization, applied to bladder and colon cancer data sets. Cancer Research 64 5245-5250.

Angrisano T, Lembo F, Pero R, Natale F, Fusco A, Avvedimento VE, Bruni CB \& Chiariotti L 2006 TACC3 mediates the association of MBD2 with histone acetyltransferases and relieves transcriptional repression of methylated promoters. Nucleic Acids Research 34 364-372.

Bazett-Jones DP, Cote J, Landel CC, Peterson CL \& Workman JL 1999 The SWI/SNF complex creates loop domains in DNA and polynucleosome arrays and can disrupt DNA-histone contacts within these domains. Molecular and Cellular Biology 19 1470-1478.

Benjamini Y \& Hochberg Y 1995 Controlling the false discovery rate - a practical and powerful approach to multiple testing. Journal of the Royal Statistical Society. Series B $\mathbf{5 7}$ 289-300.

Bhattacharya SK, Ramchandani S, Cervoni N \& Szyf M 1999 A mammalian protein with specific demethylase activity for mCpG DNA. Nature 397 579-583.

Bolstad BM, Irizarry RA, Astrand M \& Speed TP 2003 A comparison of normalization methods for high density oligonucleotide array data based on variance and bias. Bioinformatics 19 185-193.

Brazma A, Hingamp P, Quackenbush J, Sherlock G, Spellman P, Stoeckert C, Aach J, Ansorge W \& Ball CA et al. 2001 Minimum information about a microarray experiment (MIAME) - toward standards for microarray data. Nature Genetics 29 365-371.

Bultman SJ, Gebuhr TC, Pan H, Svoboda P, Schultz RM \& Magnuson T 2006 Maternal BRG1 regulates zygotic genome activation in the mouse. Genes and Development 20 1744-1754.

Carlson LL, Page AW \& Bestor TH 1992 Properties and localization of DNA methyltransferase in preimplantation mouse embryos: implications for genomic imprinting. Genes and Development 6 2536-2541.

Cervoni N \& Szyf M 2001 Demethylase activity is directed by histone acetylation. Journal of Biological Chemistry 276 40778-40787.

Cervoni N, Bhattacharya S \& Szyf M 1999 DNA demethylase is a processive enzyme. Journal of Biological Chemistry 274 8363-8366.

Cervoni N, Detich N, Seo SB, Chakravarti D \& Szyf M 2002 The oncoprotein Set/TAF-1beta, an inhibitor of histone acetyltransferase, inhibits active demethylation of DNA, integrating DNA methylation and transcriptional silencing. Journal of Biological Chemistry 277 25026-25031.

Cloos PA, Christensen J, Agger K, Maiolica A, Rappsilber J, Antal T, Hansen KH \& Helin K 2006 The putative oncogene GASC1 demethylates tri- and dimethylated lysine 9 on histone H3. Nature 442 307-311.

Detich N, Theberge J \& Szyf M 2002 Promoter-specific activation and demethylation by MBD2/demethylase. Journal of Biological Chemistry 277 35791-35794.

Detich N, Bovenzi V \& Szyf M 2003 Valproate induces replicationindependent active DNA demethylation. Journal of Biological Chemistry 278 27586-27592.

Fulka J Jr, Martinez F, Tepla O, Mrazek M \& Tesarik J 2002 Somatic and embryonic cell nucleus transfer into intact and enucleated immature mouse oocytes. Human Reproduction 17 2160-2164.

Gao S, Gasparrini B, McGarry M, Ferrier T, Fletcher J, Harkness L, De Sousa P \& Wilmut I 2002 Germinal vesicle material is essential for nucleus remodeling after nuclear transfer. Biology of Reproduction $\mathbf{6 7}$ 928-934.

Goto T, Jones GM, Lolatgis N, Pera MF, Trounson AO \& Monk M 2002 Identification and characterisation of known and novel transcripts expressed during the final stages of human oocyte maturation. Molecular Reproduction and Development 62 13-28.

Gurdon JB 2006 From nuclear transfer to nuclear reprogramming: the reversal of cell differentiation. Annual Review of Cell and Developmental Biology 22 1-22.

Hansis C, Barreto G, Maltry N \& Niehrs C 2004 Nuclear reprogramming of human somatic cells by xenopus egg extract requires BRG1. Current Biology 14 1475-1480. 
Hendrich B \& Bird A 1998 Identification and characterization of a family of mammalian methyl-CpG binding proteins. Molecular and Cellular Biology 18 6538-6547.

Irizarry RA, Bolstad BM, Collin F, Cope LM, Hobbs B \& Speed TP 2003 a Summaries of affymetrix genechip probe level data. Nucleic Acids Research 31 e15.

Irizarry RA, Hobbs B, Collin F, Beazer-Barclay YD, Antonellis KJ, Scherf U \& Speed TP 2003b Exploration, normalization, and summaries of high density oligonucleotide array probe level data. Biostatistics 4 249-264.

Kingston RE \& Narlikar GJ 1999 ATP-dependent remodeling and acetylation as regulators of chromatin fluidity. Genes and Development 13 2339-2352.

Leong HS, Yates T, Wilson C \& Miller CJ 2005 ADAPT: a database of affymetrix probesets and transcripts. Bioinformatics $212552-2553$.

Lequarre AS, Traverso JM, Marchandise J \& Donnay I 2004 Poly(A) RNA is reduced by half during bovine oocyte maturation but increases when meiotic arrest is maintained with CDK inhibitors. Biology of Reproduction 71 425-431.

Li C \& Wong WH 2003 DNA-chip analyzer (dChip). In The Analysis of Gene Expression Data: Methods and Software, pp 120-141. Eds G Parmigiani, ES Garrett, RA Irizarry \& SL Zeger. New York: Springer.

Mayer W, Niveleau A, Walter J, Fundele R \& Haaf T 2000 Demethylation of the zygotic paternal genome. Nature 403 501-502.

Mertineit C, Yoder JA, Taketo T, Laird DW, Trasler JM \& Bestor TH 1998 Sex-specific exons control DNA methyltransferase in mammalian germ cells. Development 125 889-897.

Milutinovic S, D'Alessio AC, Detich N \& Szyf M 2006 Valproate induces widespread epigenetic reprogramming which involves demethylation of specific genes. Carcinogenesis 28 560-571.

El Mouatassim S, Guerin P \& Menezo Y 1999 Expression of genes encoding antioxidant enzymes in human and mouse oocytes during the final stages of maturation. Molecular Human Reproduction 5 720-725.

Nan X, Ng HH, Johnson CA, Laherty CD, Turner BM, Eisenman RN \& Bird A 1998 Transcriptional repression by the methyl-CpG-binding protein MeCP2 involves a histone deacetylase complex. Nature 393 386-389.

Ng HH, Zhang Y, Hendrich B, Johnson CA, Turner BM, ErdjumentBromage H, Tempst P, Reinberg D \& Bird A 1999 MBD2 is a transcriptional repressor belonging to the MeCP1 histone deacetylase complex. Nature Genetics 23 58-61.

Oliveri RS 2007 Epigenetic dedifferentiation of somatic cells into pluripotency - cellular alchemy in the age of regenerative medicine? Regenerative Medicine [in press].

Oswald J, Engemann S, Lane N, Mayer W, Olek A, Fundele R, Dean W, Reik W \& Walter J 2000 Active demethylation of the paternal genome in the mouse zygote. Current Biology 10 475-478.

Pan H, O'brien MJ, Wigglesworth K, Eppig JJ \& Schultz RM 2005 Transcript profiling during mouse oocyte development and the effect of gonadotropin priming and development in vitro. Developmental Biology 286 493-506.

Peccoud J \& Jacob C 1996 Theoretical uncertainty of measurements using quantitative polymerase chain reaction. Biophysical Journal 71 101-108.

Pfaffl MW 2001 A new mathematical model for relative quantification in real-time RT-PCR. Nucleic Acids Research 29 e45.

Pollard KS, Dudoit S, van der Laan MJ 2004 Multiple testing procedures: R multtest package and applications to genomics. U.C. Berkeley Division of Biostatistics Working Paper Series. Working Paper 164 (http://www.bepress.com/ucbbiostat/paper164).

Ramchandani S, Bhattacharya SK, Cervoni N \& Szyf M 1999 DNA methylation is a reversible biological signal. PNAS 96 6107-6112.

Ratnam S, Mertineit C, Ding F, Howell CY, Clarke HJ, Bestor TH, Chaillet JR \& Trasler JM 2002 Dynamics of Dnmt1 methyltransferase expression and intracellular localization during oogenesis and preimplantation development. Developmental Biology 245 304-314.

Robertson KD, Ait-Si-Ali S, Yokochi T, Wade PA, Jones PL \& Wolffe AP 2000 DNMT1 forms a complex with Rb, E2F1 and HDAC1 and represses transcription from E2F-responsive promoters. Nature Genetics 25 338-342.
Rountree MR, Bachman KE \& Baylin SB 2000 DNMT1 binds HDAC2 and a new co-repressor, DMAP1, to form a complex at replication foci. Nature Genetics 25 269-277.

Rozen S \& Skaletsky HJ 2000 Primer3 on the WWW for general users and for biologist programmers. In Bioinformatics Methods and Protocols: Methods in Molecular Biology, pp 265-386. Eds S Krawetz \& S Misener. Totowa, NJ: Humana Press.

Santos F, Hendrich B, Reik W \& Dean W 2002 Dynamic reprogramming of DNA methylation in the early mouse embryo. Developmental Biology 241 172-182.

Shi Y, Lan F, Matson C, Mulligan P, Whetstine JR, Cole PA, Casero RA \& Shi Y 2004 Histone demethylation mediated by the nuclear amine oxidase homolog LSD1. Cell 119 941-953.

Simonsson S \& Gurdon J 2004 DNA demethylation is necessary for the epigenetic reprogramming of somatic cell nuclei. Nature Cell Biology $\mathbf{6}$ 984-990.

Spinaci M, Seren E \& Mattioli M 2004 Maternal chromatin remodeling during maturation and after fertilization in mouse oocytes. Molecular Reproduction and Development $69215-221$.

Ståhlberg A, Kubista M \& Pfaffl M 2004 Comparison of reverse transcriptases in gene expression analysis. Clinical Chemistry $\mathbf{5 0}$ 1678-1680.

Su YQ, Sugiura K, Woo Y, Wigglesworth K, Kamdar S, Affourtit J \& Eppig JJ 2007 Selective degradation of transcripts during meiotic maturation of mouse oocytes. Developmental Biology 302 104-117.

Szyf M, Eliasson L, Mann V, Klein G \& Razin A 1985 Cellular and viral DNA hypomethylation associated with induction of Epstein-Barr virus lytic cycle. PNAS 82 8090-8094.

Tanaka M, Hennebold JD, Macfarlane J \& Adashi EY 2001 A mammalian oocyte-specific linker histone gene H1oo: homology with the genes for the oocyte-specific cleavage stage histone (cs-H1) of sea urchin and the B4/H1M histone of the frog. Development 128 655-664.

Tesarik J, Martinez F, Rienzi L, Ubaldi F, lacobelli M, Mendoza C \& Greco E 2003 Microfilament disruption is required for enucleation and nuclear transfer in germinal vesicle but not metaphase II human oocytes. Fertility and Sterility 79 677-681.

Turner BM 2001 Chromatin and Gene Regulation. Molecular Mechanisms in Epigenetics, Oxford: Blackwell Science.

Vassena R, Dee SR \& Latham KE 2005 Species-dependent expression patterns of DNA methyltransferase genes in mammalian oocytes and preimplantation embryos. Molecular Reproduction and Development 72 430-436.

Wade PA, Gegonne A, Jones PL, Ballestar E, Aubry F \& Wolffe AP 1999 Mi-2 complex couples DNA methylation to chromatin remodelling and histone deacetylation. Nature Genetics 23 62-66.

Wang X \& Seed B 2003 A PCR primer bank for quantitative gene expression analysis. Nucleic Acids Research 31 e154.

Whitehouse I, Flaus A, Cairns BR, White MF, Workman JL \& OwenHughes T 1999 Nucleosome mobilization catalysed by the yeast SWI/SNF complex. Nature 400 784-787.

Wilmut I, Schnieke AE, McWhir J, Kind AJ \& Campbell KH 1997 Viable offspring derived from fetal and adult mammalian cells. Nature 385 810-813.

Wilson CL \& Miller CJ 2005 Simpleaffy: a BioConductor package for affymetrix quality control and data analysis. Bioinformatics 21 3683-3685.

Wu Z, Irizarry RA, Gentleman R, Martinez-Murillo F \& Spencer F 2004 A model based background adjustment for oligonucleotide expression arrays. Journal of the American Statistical Association 99 909-917.

Zhang LS, Zhang KY, Yao LJ, Liu SZ, Yang CX, Zhong ZS, Zheng YL, Sun QY \& Chen DY 2004 Somatic nucleus remodelling in immature and mature Rassir oocyte cytoplasm. Zygote 12 179-184.

Received 6 November 2006

First decision 4 December 2006

Revised manuscript received 8 March 2007

Accepted 16 March 2007 